\title{
Improved Extraction Repeatability and Spectral Reproducibility for Liquid Extraction Surface Analysis-Mass Spectrometry Using Superhydrophobic-Superhydrophilic Patterning
}

\author{
Joris Meurs, ${ }^{\dagger}$ Morgan R. Alexander, ${ }^{\dagger}$ Pavel A. Levkin, ${ }^{\ddagger}, \odot$ Simon Widmaier, ${ }^{\ddagger}$ Josephine Bunch,
} David A. Barrett, ${ }^{\dagger}$ and Dong-Hyun Kim* ${ }^{\dagger} \dagger$

\begin{abstract}
${ }^{\dagger}$ Advanced Materials and Healthcare Technology Division, School of Pharmacy, University of Nottingham, University Park, Nottingham, NG7 2RD, United Kingdom

${ }^{\ddagger}$ Institute of Toxicology and Genetics, Karlsruhe Institute of Technology (KIT), Hermann-von-Helmholtz Platz 1, Eggenstein-Leopoldshafen, 76344, Germany

${ }^{\S}$ Institute of Organic Chemistry, Karlsruhe Institute of Technology (KIT), Karlsruhe, 76131, Germany

"National Centre of Excellence in Mass Spectrometry Imaging, National Physical Laboratory, Hampton Road, Teddington, Middlesex TW11 0LW, United Kingdom
\end{abstract}

Supporting Information

\begin{abstract}
A major problem limiting reproducible use of liquid extraction surface analysis (LESA) array sampling of dried surface-deposited liquid samples is the unwanted spread of extraction solvent beyond the dried sample limits, resulting in unreliable data. Here, we explore the use of the Droplet Microarray (DMA), which consists of an array of superhydrophilic spots bordered by a superhydrophobic material giving the potential to confine both the sample spot and the LESA extraction solvent in a defined area. We investigated the DMA method in comparison with a standard glass substrate using LESA analysis of a mixture of biologically relevant

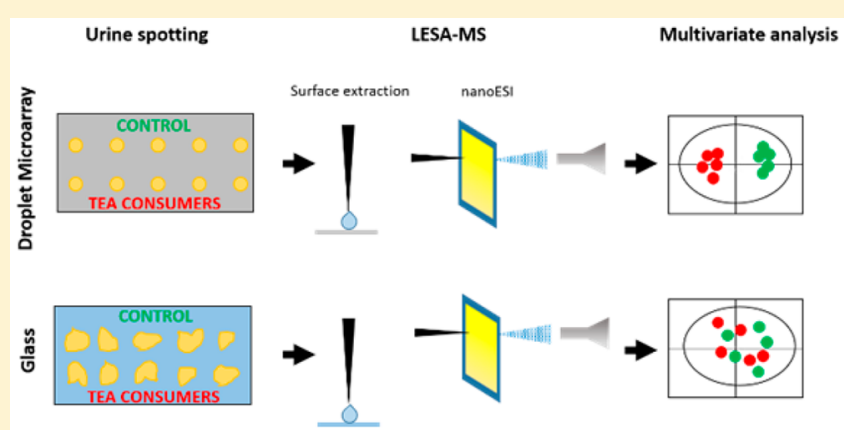
compounds with a wide mass range and different physicochemical properties. The optimized DMA method was subsequently applied to urine samples from a human intervention study. Relative standard deviations for the signal intensities were all reduced at least 3-fold when performing LESA-MS on the DMA surface compared with a standard glass surface. Principal component analysis revealed more tight clusters indicating improved spectral reproducibility for a human urine sample extracted from the DMA compared to glass. Lastly, in urine samples from an intervention study, more significant ions (145) were identified when using LESA-MS spectra of control and test urine extracted from the DMA. We demonstrate that DMA provides a surface-assisted LESA-MS method delivering significant improvement of the surface extraction repeatability leading to the acquisition of more robust and higher quality data. The DMA shows potential to be used for LESA-MS for controlled and reproducible surface extraction and for acquisition of high quality, qualitative data in a high-throughput manner.
\end{abstract}

\begin{abstract}
T iquid extraction surface analysis-mass spectrometry (LESA-MS) is an ambient mass spectrometry technique in which analytes are extracted from a surface by the formation of a liquid microjunction between the extraction solvent and the surface. ${ }^{1}$ This technique has already been widely applied for the analysis of proteins, ${ }^{2}$ metabolites, ${ }^{3}$ drugs, ${ }^{3}$ and lipids ${ }^{4}$ in a wide range of matrices and on various surface substrates. One of the main limitations of LESA-MS is poor repetitive sampling due to spreading of the probe solvent on the surface of interest. ${ }^{4}$ Irregular spreading of the extraction solvent can potentially lead to poor reproducibility of the reaspirated solvent volume ${ }^{4}$ and therefore may introduce undesired variance between spectra. A number of groups have limited the spreading of the probe solvent by using electrodes ${ }^{5}$ or applying pressure on top of the solvent. ${ }^{4}$ Another way of
\end{abstract}

limiting solvent spread is contact LESA $^{6}$ or performing laser ablation rather than liquid extraction. ${ }^{7}$

It already has been observed that the spreading of the extraction solvent on hydrophilic surfaces is substantial ${ }^{6}$ and that the use of nonpolar solvents such as chloroform dramatically increases the solvent spread. ${ }^{4}$ When the surface itself is not part of the system and is simply there to support a spot of liquid analyte, the analytical performance of liquid surface sampling could be enhanced by the judicious choice of the surface properties to vastly improve spatial confinement of

Received: March 2, 2018

Accepted: April 27, 2018

Published: April 27, 2018 
both sample deposition and the application of extraction solvent. Van Berkel et al. reported a reproducibility ranging between $10 \%$ and $34 \%$ for different lysozyme concentrations on Teflon-masked microscope slides analyzed by a continuous surface sampling probe. $^{8}$

Hence, repeatable probe solvent recovery as well as extraction efficiency could be optimized by designing a substrate surface, which confines both the liquid sample and the LESA extraction solvent within a consistent area. We propose that these requirements can be met by using a platform for high-throughput cell screening called the Droplet Microarray (DMA; http://www.aquarray.com/). The DMA consists of multiple superhydrophilic spots bordered by a superhydrophobic material produced by chemically modifying a polymer surface. ${ }^{9,10}$ These surface properties are of interest for LESA to assist in obtaining reproducible analyte spot deposition and extraction solvent coverage of the spots. The superhydrophilic part guides the solvent along the surface while the superhydrophobic material has a low surface tension and is therefore difficult to wet. ${ }^{11}$ This will limit the deposited sample as well as the extraction solvent from further spreading. Besides the surface chemistry, the use of the DMA for LESA is of interest due to its high-throughput design. Here, we compare the DMA high-throughput array to a standard glass microscope slide, which is often used as a substrate for LESA-MS, 3,4,7,12-22 to test the hypothesis that confining the sample as well as the extraction solvent in a defined area assists in obtaining repeatable solvent recovery leading to improved spectral reproducibility and multivariate modeling.

\section{MATERIALS AND METHODS}

Chemicals. Methanol, formic acid, and water were all purchased as MS grade (CHROMASOLV) from Sigma-Aldrich (Gillingham, UK). Taurine, diphenhydramine $\mathrm{HCl}$, and Rhodamine 6G were purchased from Acros Organics (Geel, Belgium). L-Arginine, raffinose pentahydrate, hemin, and vitamin B12 were bought at Sigma-Aldrich (Gillingham, UK). Sodium hydroxide was purchased from VWR International (Leuven, Belgium).

Sample Preparation. Standard Mixture. Individual stock solutions of taurine, L-arginine, diphenhydramine, Rhodamine $6 \mathrm{G}$, raffinose (all $10 \mathrm{mM})$, and vitamin $\mathrm{B} 12(1 \mathrm{mM})$ were freshly prepared in deionized water (18.2 $\mathrm{M} \Omega$; ELGA PureLab). A $10 \mathrm{mM}$ stock solution of hemin was prepared in $50 \mathrm{mM} \mathrm{NaOH}$. A mixture containing all standards was created with a working concentration of $10 \mu \mathrm{M}$ in deionized water for all compounds. Ten spots of $2 \mu \mathrm{L}$ of standard mixture were manually pipetted onto the DMA (2.8 mm hydrophilic circular spots, $1.7 \mathrm{~mm}$ superhydrophobic borders; Aquarray, Karlsruhe, Germany; Supplementary File 1) as well as a glass slide and allowed to air dry.

Urine Samples. The study design was authorized by University of Nottingham Ethical Committee of the School of Pharmacy, University of Nottingham (reference number: 021-2016). Control urine was collected without the use of preservatives from four healthy male volunteers prior to tea consumption. Then, the same volunteers consumed $200 \mathrm{~mL}$ of brewed Earl Gray tea with water (Twinings, R. Twining \& Company Limited, UK). Urine samples were collected $2 \mathrm{~h}$ after consumption and stored immediately in a $-80{ }^{\circ} \mathrm{C}$ freezer. All urine samples were thawed on ice and then briefly vortexed. Urine samples were manually pipetted $(2 \mu \mathrm{L})$ on individual superhydrophilic spots as well as onto a clean glass slide. Samples were allowed to air dry prior to analysis.

Liquid Extraction Surface Analysis-Mass Spectrometry (LESA-MS). A LESA extraction solvent was prepared containing methanol, water, and formic acid in a volume ratio of, respectively, 70:30:0.1. ${ }^{3}$ Slides were placed onto the LESA universal plate holder and scanned with an Epson V300 scanner, and coordinates of sample locations were selected with LESA Points software (Advion Biosciences, Inc., Ithaca, USA). After that, LESA was performed using the TriVersa Nanomate (Advion Biosciences, Inc., Ithaca, USA). The extraction solvent $(5 \mu \mathrm{L})$ was aspirated from the solvent reservoir from which 3 $\mu \mathrm{L}$ was dispensed onto the substrate surface. After $5 \mathrm{~s}, 3.5 \mu \mathrm{L}$ was re-aspirated into the tip and, after a delay of $10 \mathrm{~s}$, the tip was directed to a nanoESI chip. Ionization was performed at $1.45 \mathrm{kV}$ with 0.3 psi back pressure. ${ }^{3}$ Data was acquired for 1.5 min in electrospray positive ionization mode on an Exactive Orbitrap mass spectrometer (Thermo Scientific, San Jose, USA) using a scan range of $m / z 100-1500$. The resolution was set to 100000 at $\mathrm{m} / z 400$. Maximum injection was set to 1000 $\mathrm{ms}$; the AGC target was set to $1 \times 10^{6}$, and every scan consisted of 1 microscan. The capillary temperature was set to $250{ }^{\circ} \mathrm{C}$, and the declustering potential, skimmer voltage, and capillary voltage were, respectively, set to 125,20 , and $80 \mathrm{~V}$.

Data Analysis. Average scans were generated in Xcalibur v2.1 (Thermo Scientific, San Jose, USA) and exported as .RAW files. These files were converted to .mzXML files using ProteoWizard (http://proteowizard.sourceforge.net/). Converted files were further processed using a combination of SpectralAnalysis $^{23}$ and an in-house MATLAB script (R2016b, The MathWorks, Inc.).

For the standard mixture, signal intensities of the most abundant adduct ion were selected, log transformed, and normalized using the ratio of the sample's total ion count (TIC) and the class TIC average. Relative standard deviations (RSD) of the signal intensity were calculated per standard for both surfaces and statistically compared using Forkman's Ftest $^{24}(\alpha=0.05)$.

For the urine samples, spectral data were background subtracted and Savitzky-Golay smoothed, ${ }^{25}$ and a correction was applied for signal intensity loss due to smoothing by multiplying the smoothed data with a factor. This multiplication factor was calculated by taking the ratio of the original base peak intensity and the smoothed base peak intensity (see Supplementary File 2 for an example). Subsequently, spectra were $\log$ transformed and TIC normalized. Spectral reproducibility was checked using peaks with an $80 \%$ detection rate ${ }^{26}$ (threshold intensity: $1 \times 10^{3}$ ) within all analytical replicates $(n$ $=5$ ) of a control urine sample.

Metabolic profiling was done using the same preprocessing steps except peaks were only included at a class detection rate of $75 \%$. Missing values were replaced by using mean imputation $^{27}$ per class. After preprocessing, counting of unique and significant ions between classes was performed. Comparison of TIC normalized peak intensities was done using Student's $t$ test at a significance level of 0.05 , and $p$-values were corrected on the basis of a false discovery rate (FDR) using the Benjamini-Hochberg procedure. ${ }^{28}$ After, the data were Pareto scaled $^{29}$ and subjected to principal component analysis (PCA).

\section{RESULTS AND DISCUSSION}

Extraction Repeatability and Spectral Reproducibility. Ten dried spots of standard analyte mixture on glass and DMA 
Table 1. Comparison of Relative Standard Deviations of Signal Intensities per Compound between Glass and DMA

\begin{tabular}{|c|c|c|c|c|c|}
\hline compound & adduct & $m / z$ & RSD glass (\%) & RSD DMA (\%) & $p$-value \\
\hline Taurine & {$[\mathrm{M}+\mathrm{Na}]^{+}$} & 148.0044 & 25.3 & 7.6 & 0.015 \\
\hline L-Arginine & {$[\mathrm{M}+\mathrm{Na}]^{+}$} & 197.1015 & 23.1 & 7.3 & 0.020 \\
\hline Diphenhydramine & {$[\mathrm{M}+\mathrm{H}]^{+}$} & 256.1701 & 20.1 & 5.1 & $4.0 \times 10^{-4}$ \\
\hline Rhodamine $6 \mathrm{G}$ & {$[\mathrm{M}-\mathrm{Cl}]^{+}$} & 443.2335 & 20.0 & 5.1 & $4.3 \times 10^{-4}$ \\
\hline Raffinose & {$[\mathrm{M}+\mathrm{Na}]^{+}$} & 527.1588 & 21.7 & 7.4 & 0.0034 \\
\hline Hemin & {$[\mathrm{M}-\mathrm{Cl}]^{+}$} & 616.1773 & 21.4 & 5.6 & $7.7 \times 10^{-4}$ \\
\hline Vitamin B12 & {$[\mathrm{M}+2 \mathrm{Na}]^{+}$} & 700.2735 & 26.6 & 7.9 & 0.0056 \\
\hline
\end{tabular}
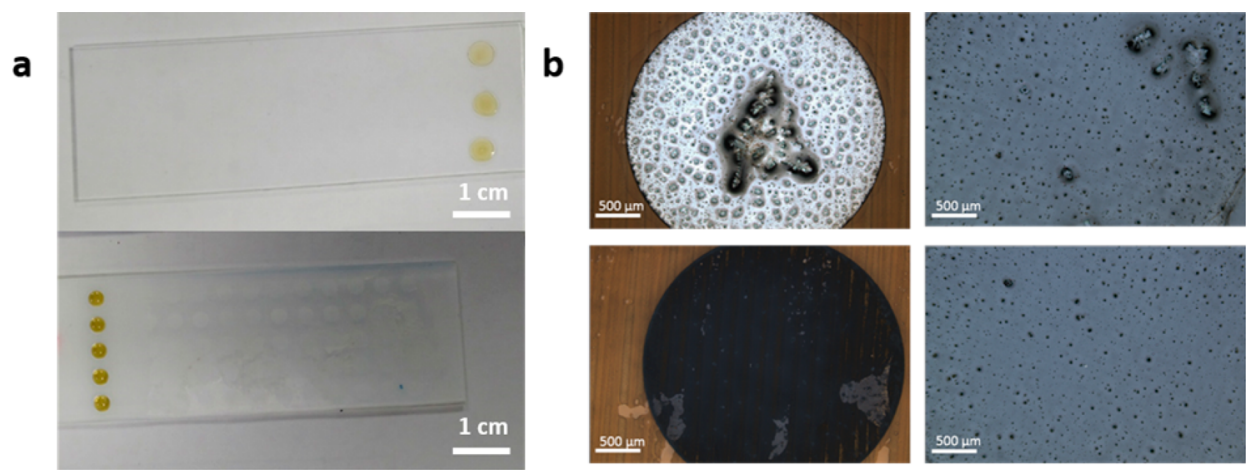

Figure 1. Depositing and extraction of samples from the Droplet Microarray and glass surface. (a) $10 \mathrm{mM}$ hemin in $50 \mathrm{mM} \mathrm{NaOH}$ manually pipetted onto glass (top) and Droplet Microarray (bottom). Sample volume: $3 \mu \mathrm{L}$; spot size DMA: $\sim 2.8 \mathrm{~mm}$; spot size glass: $\sim 4.0 \mathrm{~mm}$. (b) Microscope image (Optical Profile, Zeta Instruments; $5 \times$ magnification) of dried urine on Droplet Microarray (left) and glass (right). Images taken before (top) and after extraction (bottom). Visually higher efficient extraction from DMA. Sample volume: $2 \mu \mathrm{L}$.
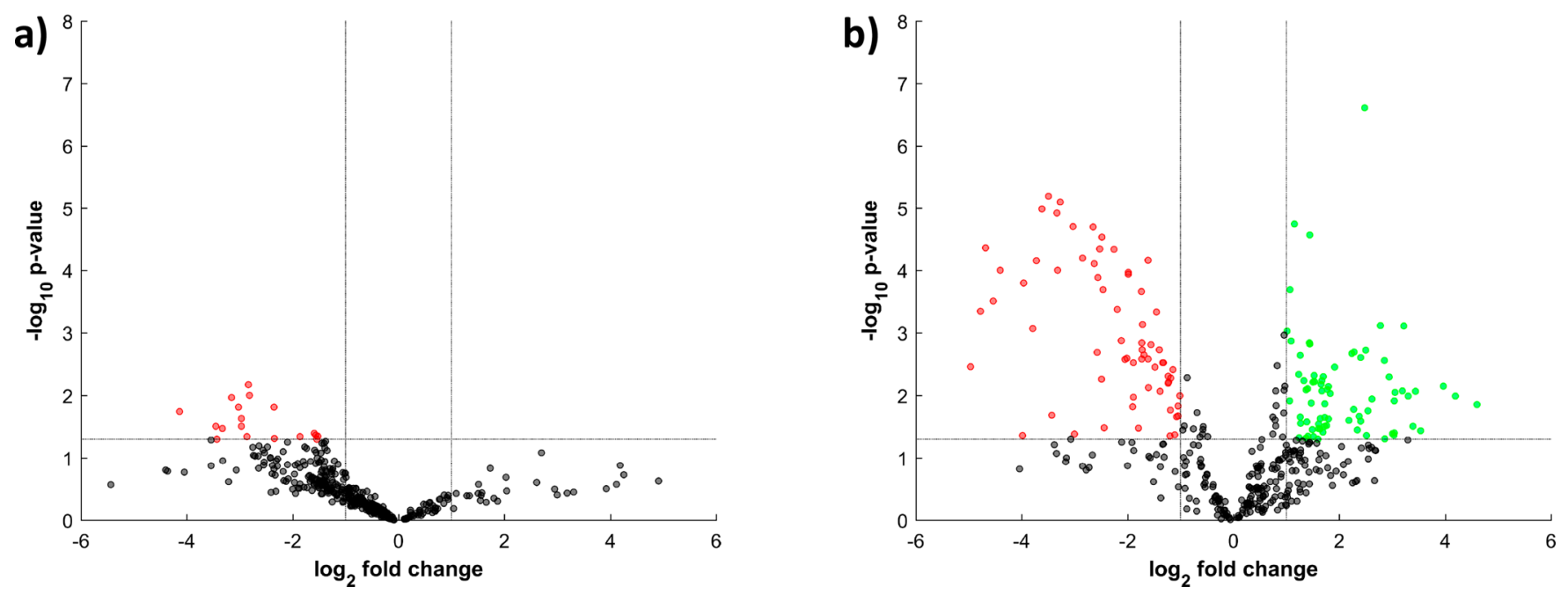

Figure 2. Volcano plots for common ions between urine samples before and after tea consumption. (a) Urine extracted from glass. (b) Urine extracted from DMA. Green and red data points indicate ions, which are, respectively, increasing and decreasing significantly $(p<0.05)$ and at least 2-fold in intensity.

were analyzed using LESA-MS, and RSDs were calculated for the most abundant adduct ion for each standard (Table 1). All RSDs were below $8 \%$ when extracted from the DMA while extraction from glass resulted in RSDs above $20 \%$ which is higher than the limit of the acceptable precision (15\%) for an analytical method. ${ }^{30}$

Analytical replicates of a human urine control sample $(n=5)$ were dispensed at five locations on glass as well as on five DMA spots, and the dried urine spots were analyzed with LESA-MS (Supplementary File 3). Data were subjected to peak picking ( $\geq 80 \%$ detection rate) and subsequently normalized to the TIC and log transformed to compare the deviations per peak intensity between both surfaces. It was observed that the RSD for each ion was reduced (Supplementary File 3), indicating better spectral reproducibility using the DMA as a substrate compared with glass. The high degree of spectral similarity between replicate samples can be related to the confinement of both the sample and the extraction solvent within a defined area (Figure 1), which increases the extraction repeatability. The superhydrophilic spots allow increased wetting of the surface $^{31}$ thus improving analyte deposition uniformity and resulting in increased LESA solvent extraction efficiency. Further, confining the extraction solvent within a defined area would be expected to result in a more stable microjunction formed between the tip and surface and hence explain the improved analytical performance. Therefore, the use of the 

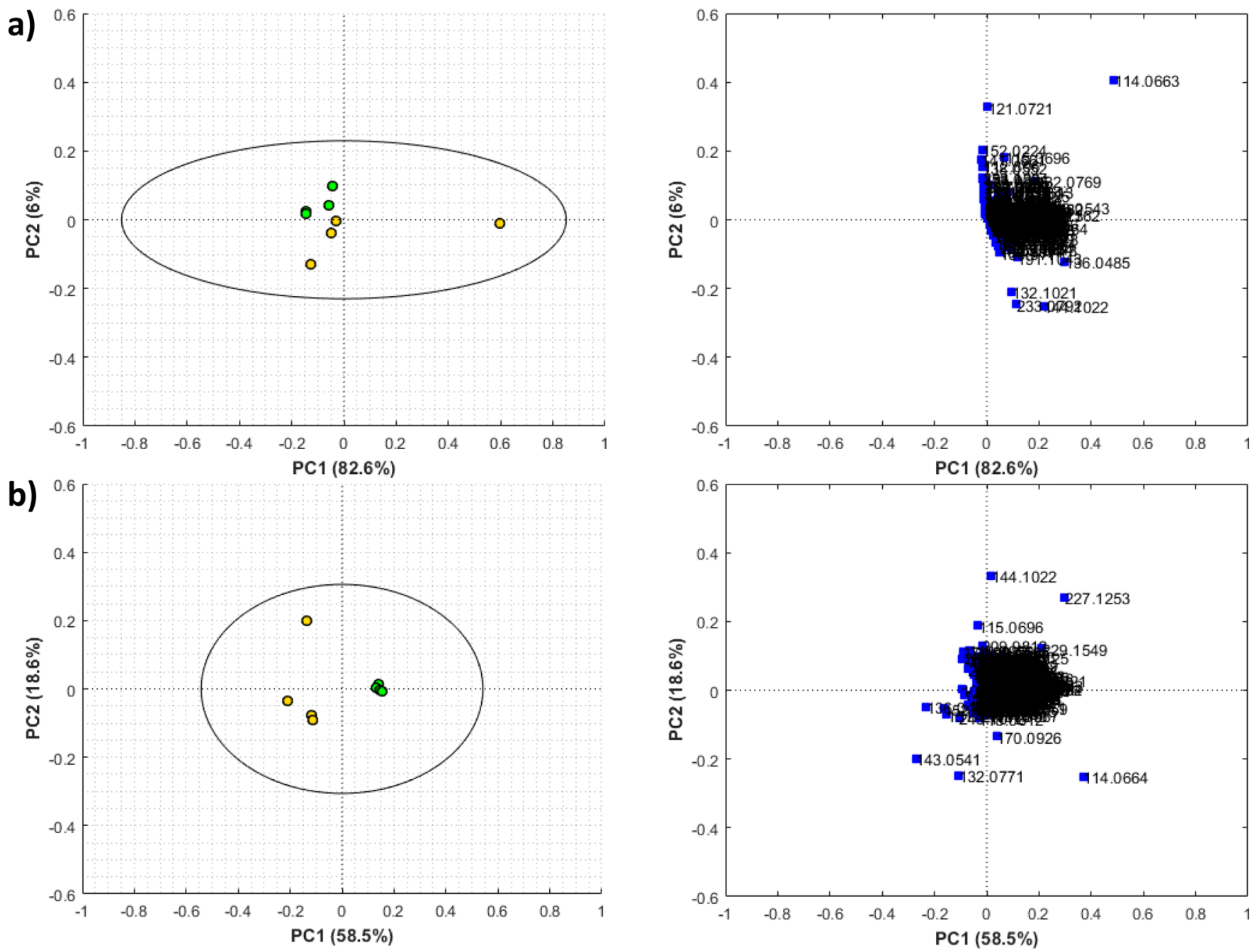

Figure 3. PCA scores and loadings plot for ions ( $\geq 75 \%$ class detection) found in urine before (green) and after tea consumption (yellow) extracted from (a) glass $\left(R^{2}: 0.7184 ; Q^{2}:-0.1551\right)$ and (b) DMA $\left(R^{2}: 0.9609 ; Q^{2}: 0.8938\right)$. Data was TIC normalized and Pareto scaled.

DMA with hydrophilic spots permits longer extraction times without the loss of solvent, with a benefit for enhancing the extraction efficiency. ${ }^{32}$

Metabolic Profiling. Control $(n=4)$ and tea consumers' $(n=4)$ urine samples were spotted onto glass and the DMA and, after drying, subjected to LESA-MS analysis. The collected spectra were analyzed in MATLAB. On average, 544 and 1343 features were found in the urine samples before and after tea consumption on the DMA, respectively. In contrast, LESA-MS analysis of urine before and after tea consumption on a glass surface resulted in, respectively, 941 and 806 features on average. At a $75 \%$ class detection rate, more unique ions were found in urine after tea consumption using the DMA $(n=340)$ as substrate compared to glass $(n=12)$. A total of 395 and 453 common ions were found between urine samples before and after tea consumption on DMA and glass, respectively. Peak intensities were compared using a Student's $t$ test $(\alpha=0.05)$, and $p$-values were FDR corrected. This resulted in 163 significant ions for urine samples extracted from the DMA while only 18 significant ions were found when glass was used as substrate (Figure 2). Subjecting the data to PCA after TIC normalization and Pareto scaling showed better clustering for the control samples in the scores plot when the DMA was used as substrate $\left(R^{2}: 0.9609 ; Q^{2}: 0.8938\right.$; Figure 3$)$. In contrast, the scores plot for data acquired from glass showed no separation between the urine groups $\left(R^{2}: 0.7184 ; Q^{2}:-0.1551\right)$. Again, the substantial increase of the number of significant ions as well as the improvement in multivariate modeling can be related to enhanced reproducibility of the solvent recovery and extraction efficiency. These findings show potential of using the DMA in combination with LESA-MS as an alternative to conventional methods for profiling of biological samples. Compared to direct infusion MS, ion suppression due to a high nonvolatile salt content in a biological sample ${ }^{33}$ could be reduced due to the dilution effect of the sample in the LESA extraction solvent. Further, the reduced run time and solvent consumption could favor LESA-MS over liquid chromatography-mass spectrometry (LC-MS).

\section{CONCLUSION}

We demonstrate that the DMA surface has significant advantages for consistent, high-throughput LESA-MS analysis, which we attribute to the superhydrophilic-superhydrophobic array patterning. We found that the specific DMA surface properties improved extraction repeatability and spectral reproducibility resulting in higher quality metabolic profiling. This can be of use for cost-effective and fast clinical screening/ profiling of human clinical samples such as body fluids (e.g., 
urine, blood). Moreover, increased sampling and spectral reproducibility could provide opportunities for improved quantitative analysis when suitable internal standards are available. In addition, the extraction solvent is confined within the sample area, which allows extended extraction times and provides potential benefits for the analysis of poorly soluble compounds. In conclusion, the DMA shows a huge potential for cost-effective and robust high-throughput screening/ profiling of liquid matrices using LESA-MS, since only small volumes of sample and extraction solvent are required. Furthermore, there is no risk of cross contamination during sampling and extraction, and sample data can be acquired, depending on solubility, within a short time interval.

\section{ASSOCIATED CONTENT}

\section{S Supporting Information}

The Supporting Information is available free of charge on the ACS Publications website at DOI: 10.1021/acs.analchem.8b00973.

Dimensions of an array feature on the Droplet Microarray; example of signal correction after SavitzkyGolay smoothing; LESA-MS spectra for five analytical replicates of control urine extracted from Droplet Microarray (DMA) and glass; box plots and relative standard deviations for common ions in control urine extracted from glass and Droplet Microarray (PDF)

\section{AUTHOR INFORMATION}

\section{Corresponding Author}

*E-mail: dong-hyun.kim@nottingham.ac.uk.

\section{ORCID}

Joris Meurs: 0000-0002-0288-0422

Pavel A. Levkin: 0000-0002-5975-948X

\section{Notes}

The authors declare no competing financial interest.

\section{ACKNOWLEDGMENTS}

This work was supported by the Engineering and Physical Sciences Research Council [grant number EP/N006615/1]. Further, the authors would like to thank Sergey Evseev for providing the urine samples.

\section{REFERENCES}

(1) Kertesz, V.; Van Berkel, G. J. J. Mass Spectrom. 2010, 45 (3), 252-260.

(2) Rao, W.; Celiz, A. D.; Scurr, D. J.; Alexander, M. R.; Barrett, D. A. J. Am. Soc. Mass Spectrom. 2013, 24 (12), 1927-1936.

(3) Bailey, M. J.; Randall, E. C.; Costa, C.; Salter, T. L.; Race, A. M.; de Puit, M.; Koeberg, M.; Baumert, M.; Bunch, J. Anal. Methods 2016, 8 (16), 3373-3382.

(4) Almeida, R.; Berzina, Z.; Arnspang, E. C.; Baumgart, J.; Vogt, J.; Nitsch, R.; Ejsing, C. S. Anal. Chem. 2015, 87 (3), 1749-1756.

(5) Brenton, A. G.; Godfrey, A. R. Anal. Chem. 2014, 86 (7), 33233329.

(6) Randall, E. C.; Bunch, J.; Cooper, H. J. Anal. Chem. 2014, 86

(21), 10504-10510.

(7) Lorenz, M.; Ovchinnikova, O. S.; Van Berkel, G. J. Rapid Commun. Mass Spectrom. 2014, 28 (11), 1312-1320.

(8) Van Berkel, G. J.; Ford, M. J.; Doktycz, M. J.; Kennel, S. J. Rapid Commun. Mass Spectrom. 2006, 20 (7), 1144-1152.

(9) Popova, A. A.; Schillo, S. M.; Demir, K.; Ueda, E.; NesterovMueller, A.; Levkin, P. A. Adv. Mater. 2015, 27 (35), 5217-5222.
(10) Geyer, F. L.; Ueda, E.; Liebel, U.; Grau, N.; Levkin, P. A. Angew. Chem., Int. Ed. 2011, 50 (36), 8424-8427.

(11) Valipour, M. N.; Birjandi, F. C.; Sargolzaei, J. Colloids Surf., A 2014, 448, 93-106.

(12) Kertesz, V.; Van Berkel, G. J. Bioanalysis 2013, 5 (7), 819-826.

(13) Ellis, S. R.; Ferris, C. J.; Gilmore, K. J.; Mitchell, T. W.; Blanksby, S. J.; et al. Anal. Chem. 2012, 84 (22), 9679-9683.

(14) Moench, P. A.; Catoire, A.; Glick, J.; Flarakos, J. Rapid Commun. Mass Spectrom. 2016, 30 (2), 340-342.

(15) Mikhailov, V. A.; Griffiths, R. L.; Cooper, H. J. Int. J. Mass Spectrom. 2017, 420, 43-50.

(16) Tomlinson, L.; Fuchser, J.; Fütterer, A.; Baumert, M.; Hassall, D. G.; West, A.; Marshall, P. S. Rapid Commun. Mass Spectrom. 2014, 28 (9), 995-1003.

(17) Sarsby, J.; Martin, N. J.; Lalor, P. F.; Bunch, J.; Cooper, H. J. J. Am. Soc. Mass Spectrom. 2014, 25 (11), 1953-1961.

(18) Griffiths, R. L.; Cooper, H. J. Anal. Chem. 2016, 88 (1), 606609 .

(19) Eikel, D.; Vavrek, M.; Smith, S.; Bason, C.; Yeh, S.; Korfmacher, W. A.; Henion, J. D. Rapid Commun. Mass Spectrom. 2011, 25 (23), $3587-3596$

(20) Hall, Z.; Ament, Z.; Wilson, C. H.; Burkhart, D. L.; Ashmore, T.; Koulman, A.; Littlewood, T.; Evan, G. I.; Griffin, J. L. Cancer Res. 2016, 76 (16), 4608-4618.

(21) Griffiths, R. L.; Creese, A. J.; Race, A. M.; Bunch, J.; Cooper, H. J. Anal. Chem. 2016, 88 (13), 6758-6766.

(22) Hall, Z.; Chu, Y.; Griffin, J. L. Anal. Chem. 2017, 89 (9), 51615170.

(23) Race, A. M.; Palmer, A. D.; Dexter, A.; Steven, R. T.; Styles, I. B.; Bunch, J. Anal. Chem. 2016, 88 (19), 9451-9458.

(24) Forkman, J. Commun. Stat. - Theory Methods 2009, 38 (2), 233251.

(25) Savitzky, A.; Golay, M. J. Anal. Chem. 1964, 36 (8), 1627-1639.

(26) Yang, J.; Zhao, X.; Lu, X.; Lin, X.; Xu, G. Front. Mol. Biosci. 2015, 2 (4), 1-9.

(27) Dray, S.; Josse, J. Plant Ecol. 2015, 216 (5), 657-667.

(28) Benjamini, Y.; Hochberg, Y. J. R. Stat. Soc. Ser. B 1995, 57 (1), 289-300.

(29) van den Berg, R. A.; Hoefsloot, H. C.; Westerhuis, J. A.; Smilde, A. K.; van der Werf, M. J. BMC Genomics 2006, 7 (1), 142.

(30) Hartmann, C.; Smeyers-Verbeke, J.; Massart, D.; McDowall, R. J. Pharm. Biomed. Anal. 1998, 17 (2), 193-218.

(31) Drelich, J.; Chibowski, E. Langmuir 2010, 26 (24), 1862118623.

(32) Van Berkel, G. J.; Kertesz, V. Rapid Commun. Mass Spectrom. 2013, 27 (12), 1329-1334.

(33) Clarke, D. J.; Campopiano, D. J. Analyst 2015, 140 (8), 26792686. 